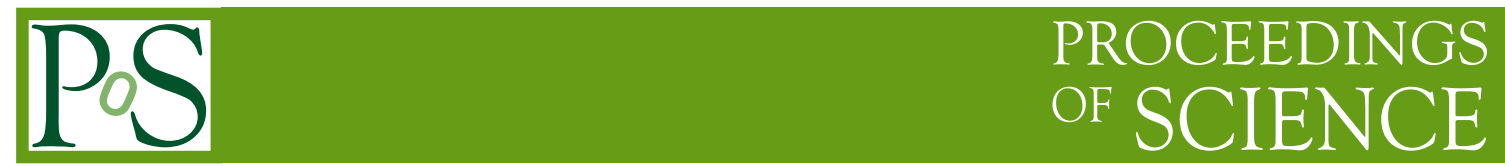

\title{
Measurement of diboson production in early LHC data
}

\author{
Vuko Brigljević * \\ Rudjer Boskovic Institute \\ E-mail: vuko.brigljevic@irb.hr
}

\begin{abstract}
We investigate the discovery potential for the $p p \rightarrow W Z^{0} \rightarrow \ell^{ \pm} v \ell^{+} \ell^{-}(\ell=e, \mu)$ process at CMS, using full detector simulation of the signal and of the various physics and instrumental backgrounds. We propose data driven methods to extract backgrounds and estimate that we can reach $5 \sigma$ significance of $W Z^{0}$ signal at CMS with less than $350 \mathrm{pb}^{-1}$ at $95 \%$ C.L..
\end{abstract}

2008 Physics at LHC

September 29 - 4 October 2008

Split, Croatia

* On behalf of the CMS Collaboration 


\section{Introduction}

The study of multiple gauge-boson production at the $\mathrm{TeV}$ scale constitutes a unique opportunity to test the Standard Model of Electroweak interactions at the highest possible energies. Production cross sections for all diboson processes, $W Z^{0}, Z^{0} Z^{0}, W W, W \gamma$ and $Z^{0} \gamma$ are relatively large and these processes are expected to be observed already with luminosities of a few hundreds inverse picobarns at the LHC.

A study of $W Z^{0}$ production in $p p$ collisions at a center of mass energy of $14 \mathrm{TeV}$ is presented in the following. The production of $W Z^{0}$ events allows probing triple gauge-boson couplings and, therefore, non-Abelian gauge symmetry of the Standard Model at energies never before attained. Any deviation of the strength of these couplings from their Standard Model expectations indicates new physics.

We consider the four leptonic final states involving electrons and muons, labeled in the following as $3 e, 2 e 1 \mu, 2 \mu 1 e$ and $3 \mu$.

We use signal and background Monte Carlo samples that are processed with the full simulation of the CMS detector [1]. The backgrounds are processes with three or more genuine leptons or misidentified jets in the final state. The largest instrumental backgrounds to all four signatures is due to misidentified jets from associated production of jets and heavy bosons and production of top quarks. Among these backgrounds, the main contribution to the $W Z^{0}$ signal is from $Z^{0}+$ jets processes. The only irreducible backgrounds to the $W Z^{0}$ final state are the $Z^{0} Z^{0}$ production with one of the leptons being mis-reconstructed or lost and $Z^{0} \gamma$ process where a photon converts and produces a genuine electron.

\section{Event Selection}

A key element for this study is an effective selection of electrons and muons. The selection criteria must have high efficiency for true isolated leptons from heavy boson decays, while at the same time effectively suppressing leptons from heavy quark decays as well as other objects misidentified as leptons.

The identification of electrons is based on matching of a charged track reconstructed in the central tracker with an energy deposition (supercluster) in the electromagnetic calorimeter (ECAL) [2]. Suppression of fake electrons is achieved by applying requirements on the shape of the ECAL energy deposition, on the matching in position and energy between the charged track and the supercluster. Muon candidates are identified by matching a track reconstructed in the muon detectors with a track reconstructed in the central tracker. To further suppress backgrounds originating mainly from genuine muons from heavy quark decays, the significance of the impact parameter in the transverse plane of a muon candidate must also be consistent with a track originating from the primary interaction point. Furthermore, requirements on calorimetric and track isolation are applied for both electron and muon candidates.

Events stemming from the three-lepton final states of $W Z^{0}$ production are collected by electron and/or muon triggers. Events are accepted if they contain at least three charged leptons, either electrons or muons, with $p_{T}>15 \mathrm{GeV}$ and $|\eta|<2.5$ (2.4) for electrons (muons). To select $W Z^{0}$ 
candidates we form all possible $Z^{0}$-boson candidates from same-flavour, opposite-charge lepton pairs.

Events are retained if the invariant mass of the $Z^{0}$ boson candidate is between 50 and $120 \mathrm{GeV}$. The event is rejected if a second $Z^{0}$ boson candidate is found with an invariant mass within $20 \mathrm{GeV}$ around the nominal $Z^{0}$ mass. If two $Z^{0}$ boson candidates are found which share one of the leptons, we select the candidate with the invariant mass closest to the nominal $Z^{0}$ mass.

After the $Z^{0}$ boson candidate is identified, we search for a third lepton in the event to be associated to the $W$ boson decay. This lepton is required to carry a transverse momentum above $20 \mathrm{GeV}$. If more than one lepton candidate is available, the one with the highest transverse momentum is chosen.

Further suppression of the background can be achieved by applying $W$ identification requirements. We define a $W$ boson candidate transverse mass as

$$
M_{T}(W)=\sqrt{2 \cdot M E T \cdot E_{\ell}\left(1-\cos \Delta \phi_{M E T, \ell}\right)}
$$

where MET is the missing transverse energy, $E_{\ell}$ is the energy of the $W$ lepton candidate, and $\triangle \phi_{M E T, \ell}$ is the azimuthal separation between the MET direction and the $W$ lepton. We require the transverse mass to be larger than $50 \mathrm{GeV}$. The event yield for the signal and background events after the full selection is summarized in Table 1.

\begin{tabular}{lccccc}
\hline \hline & all & $3 e$ & $2 e 1 \mu$ & $2 \mu 1 e$ & $3 \mu$ \\
\hline$W Z^{0}$ & $34.9 \pm 0.5$ & $7.9 \pm 0.3$ & $8.0 \pm 0.3$ & $8.9 \pm 0.3$ & $10.1 \pm 0.3$ \\
$Z^{0}+$ jets & $3.9 \pm 1.0$ & $1.9 \pm 0.8$ & $<0.1$ & $1.8 \pm 0.7$ & $0.1 \pm 0.1$ \\
bbll & $2.9 \pm 0.3$ & $1.2 \pm 0.2$ & $0.1 \pm 0.1$ & $1.3 \pm 0.2$ & $0.3 \pm 0.1$ \\
$t \bar{t}+$ jets & $2.2 \pm 0.6$ & $0.6 \pm 0.3$ & $0.6 \pm 0.3$ & $0.6 \pm 0.3$ & $0.3 \pm 0.2$ \\
$W+$ jets & $0.4 \pm 0.4$ & $0.4 \pm 0.4$ & $<0.1$ & $<0.1$ & $<0.1$ \\
$Z^{0} Z^{0}$ & $2.8 \pm 0.3$ & $0.8 \pm 0.1$ & $0.6 \pm 0.1$ & $0.7 \pm 0.1$ & $0.7 \pm 0.1$ \\
$Z^{0}+\gamma$ & $1.4 \pm 0.1$ & $0.7 \pm 0.1$ & $<0.1$ & $0.7 \pm 0.1$ & $<0.1$ \\
\hline \hline Total non genuine $Z^{0}$ bkg & $2.6 \pm 0.7$ & $1.0 \pm 0.5$ & $0.6 \pm 0.3$ & $0.6 \pm 0.3$ & $0.3 \pm 0.2$ \\
Total genuine $Z^{0}$ instrumental bkg & $6.8 \pm 1.0$ & $3.1 \pm 0.8$ & $0.1 \pm 0.1$ & $3.1 \pm 0.5$ & $0.4 \pm 0.1$ \\
Total genuine $Z^{0}$ physics bkg & $4.2 \pm 0.3$ & $1.5 \pm 0.1$ & $0.6 \pm 0.1$ & $1.4 \pm 0.1$ & $0.7 \pm 0.1$ \\
\hline \hline
\end{tabular}

Table 1: Expected number of selected events for an integrated luminosity of $300 \mathrm{pb}^{-1}$ for the signal and background with $81 \mathrm{GeV}<M_{Z}<101 \mathrm{GeV}$ obtained using MC truth information. The uncertainties are statistical.

\section{Signal Extraction}

We separate backgrounds into three categories: physics background from $Z^{0} \gamma$ and $Z^{0} Z^{0}$ production, processes without a genuine $Z^{0}$ boson from $t \bar{t}+$ jets and $W+$ jets production, and processes with a genuine $Z^{0}$ boson from $Z^{0}+$ jets production.

The physics background from $Z^{0} Z^{0}$ and $Z^{0} \gamma$ production is estimated from Monte Carlo simulation. We assign a conservative $100 \%$ uncertainty on the estimated $Z^{0} Z^{0}$ and $Z^{0} \gamma$ contribution 
due to modeling of kinematics of the decay products and the photon conversion probability. We also use the Monte Carlo simulation to estimate the background from from $t \bar{t}+$ jets and $W+$ jets processes, and we assign an additional $100 \%$ systematic uncertainty on that contribution. With increasing luminosity, this contribution can be estimated from the side-bands of the $Z^{0}$ candidate invariant mass distribution.

The remaining and also the largest instrumental background is from processes with real $Z^{0}$ boson and jets where one of the jets is misidentified as a lepton. We estimate this background in data by applying a method, commonly referred to as a "matrix method" (see for example [3]). The idea of the method is to apply a "loose" identification criteria on the lepton associated to the $W$ boson decay, after a $Z^{0}$ boson candidate is identified and count the number of observed events, $N_{\text {loose }}$. These events contain events with real isolated leptons $N_{\ell}$ and events with misidentified jets $N_{j}$ :

$$
N_{\text {loose }}=N_{\ell}+N_{j}
$$

We then apply a "tight" selection on the third lepton, the number of observed events $N_{\text {tight }}$ changes as following

$$
N_{\text {tight }}=\varepsilon_{\text {tight }} N_{\ell}+p_{\text {fake }} N_{j},
$$

where $\varepsilon_{\text {tight }}$ and $p_{\text {fake }}$ are efficiency of "tight" criteria with respect to "loose" requirements for true isolated leptons and misidentified jets, respectively. The "tight" selection uses the final selection criteria applied on the third lepton as used in the analysis, while some of these requirements are loosened for the "loose" sample.

As $N_{\text {loose }}$ and $N_{\text {tight }}$ are directly observable, to extract the number of $Z+$ jets events in the final sample, one needs to measure $\varepsilon_{\text {tight }}$ and $p_{\text {fake }}$ in control data samples. To estimate $\varepsilon_{\text {tight }}$ we apply the "tag-and-probe" method [4] using $Z^{0} \rightarrow e^{+} e^{-}$or $Z^{0} \rightarrow \mu^{+} \mu^{-}$events. The determination of $p_{\text {fake }}$ is done on a control-sample of jets misidentified as leptons. The jet composition (heavy vs light flavour, quark vs gluon jets) of the control sample must be similar to the one of the $Z^{0}+$ jets events passing the signal selection. Thus, we propose to measure $p_{\text {fake }}$ in a $W+$ jets sample which has a similar jet composition as in $Z^{0}+$ jets. The obtained $p_{\text {fake }}$ is further cross-checked with $p_{\text {fake }}$ measured in multijet events. We performed both measurements and found the $p_{\text {fake }}$ values to agree within uncertainties.

Using the values of $\varepsilon_{\text {tight }}$ and $p_{\text {fake }}$ obtained from the methods described above, we estimated the backgrounds from genuine $Z^{0}$ decays by solving Eqs. 3.1 and 3.2 for $N_{j}$. The results are given in Table 2. They agree well with the true Monte Carlo yields.

\section{Results}

The distribution of the $Z^{0}$ candidate invariant mass for all four channels combined after applying the final selection is shown in Fig. 1.

We estimated full systematic uncertainties for each of the four individual signature, they amount to $34 \%, 25 \%, 35 \%$ and $21 \%$ for the $3 e, 2 e 1 \mu, 2 \mu 1 e$ and $3 \mu$ final states, respectively. The largest contributions come from the luminosity determination and the requirement on $M_{T}(W)$. It is comparable to the statistical uncertainty which is roughly $30 \%$ for each channel. 


\begin{tabular}{lcccc}
\hline \hline & $3 \mathrm{e}$ & $2 \mathrm{e} 1 \mu$ & $2 \mu 1 \mathrm{e}$ & $3 \mu$ \\
\hline$N$ - ZZ -Z $\gamma-\mathrm{W}+$ jets - $t \bar{t}$ & $11.1 \pm 1.3$ & $8.2 \pm 0.9$ & $12.1 \pm 1.2$ & $10.5 \pm 0.8$ \\
\hline$N^{\text {genuine } Z}$ (matrix method) & $3.2 \pm 1.7$ & $0.6 \pm 0.8$ & $4.6 \pm 2.0$ & $0.6 \pm 0.9$ \\
\hline$N^{W Z^{0}}$ & $7.9 \pm 2.1$ & $7.6 \pm 1.2$ & $7.5 \pm 2.3$ & $10.0 \pm 1.2$ \\
\hline$W Z^{0}$ from MC & 7.9 & 8.1 & 9.0 & 10.1 \\
\hline
\end{tabular}

Table 2: Expected number of events for an integrated luminosity of $300 \mathrm{pb}^{-1}$ for the signal and estimated background for $81 \mathrm{GeV}<M_{Z}<101 \mathrm{GeV}$ using data-driven methods. Uncertainty is systematic associated with the background subtraction method only.

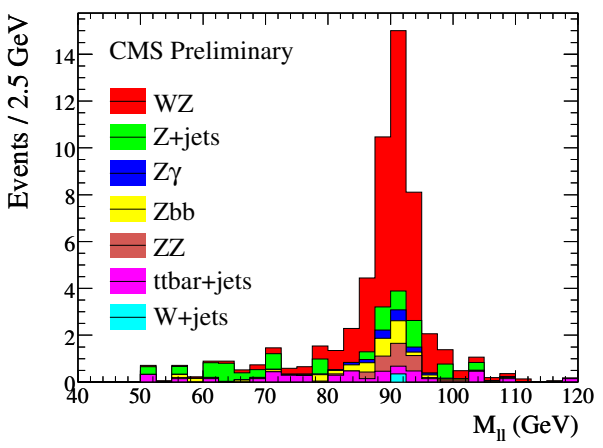

Figure 1: $Z^{0}$ candidate invariant mass for all four channels combined, normalized to integrated luminosity of $300 \mathrm{pb}^{-1}$.

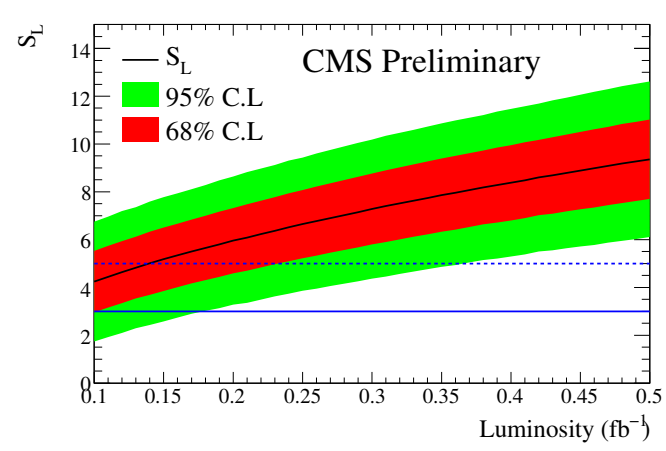

Figure 2: Expected signal significance for $W Z^{0}$ production as a function of integrated luminosity. We use a frequentist approach to estimate variation of expected signal and background events. The corresponding $68 \%$ and 95\% C.L. regions are displayed as red and green bands, respectively.

The expected signal significance $S_{L}$ of a $W Z^{0}$ production as a function of integrated luminosity for all four categories combined, after applying final selection criteria and requirement on the $Z^{0}$ invariant mass to be within $10 \mathrm{GeV}$ from the nominal $Z^{0}$ boson mass, taking into account full systematic and statistical uncertainties is shown in Fig. 2. We expect to achieve $S_{L}=5$ with less than $350 \mathrm{pb}^{-1}$ of integrated luminosity at $95 \%$ C.L..

\section{References}

[1] R. Adolphi et al. [CMS Collaboration], The CMS experiment at the CERN LHC, JINST 3 (2008) S08004

[2] S. Baffioni et al., Electron Reconstruction in CMS, CMS Note 2006-40 (2006).

[3] V. M. Abazov et al. [D0 Collaboration], $Z \gamma$ production and limits on anomalous $Z Z \gamma$ and $Z \gamma \gamma$ couplings in p $\bar{p}$ collisions at $\sqrt{s}=1.96-\mathrm{TeV}$, Phys. Lett. $\mathbf{B 6 5 3}$ (2007) 378-386.

[4] CMS Collaboration, Measuring Electron Efficiencies at CMS with Early Data, CMS PAS EGM-07-001 (2007). 